\title{
NÉMATODES DU GENRE EUCOLEUS DUJ., 1845 \\ CHEZ LES OISEAUX
}

\author{
Par Z. WASSILKOWA et L. GOUCHANSKAJA
}

Des représentants du genre Eucoleus Duj., 1845, n'ont été jusqu’à présent décrits que chez les Mammifères. A ce genre se rattachent les 3 espèces suivantes :'Eucoleus aerophilus (Creplin, 1839) des Carnivores, E. tenuis Duj., 1845 des Insectivores et E. boskakovi Schultz, 1929 des Rongeurs.

En étudiant les Capillarinés chez les Oiseaux des familles des Corvidés, Laridés et Tétraonidés, nous avons rencontré quelques espèces dont les mâles avaient une gaine spiculaire fort accusée, mais pas le moindre indice de spicules.

Nous en conclûmes que les représentants du genre Eucoleus Duj., 1845 pouvaient parasiter non seulement les Mammifères, mais aussi les Oiseaux. L'une de nous ( $Z$. Wassilkowa) a décrit ailleurs 2 espèces de ce genre chez les Corvidés et les Laridés.

D'un autre côté, en étudiant dans la bibliographie de l'espèce désignée sous le nom de Capillarina (Thominx) strumosa (Reibisch, 1893 ) et que l'une de nous avait trouvé chez un nouvel hôte, Lyrurus tetrix, nous en conclûmes que ce parasite n'avait pas non plus de spicules, tout en possédant une gaine spiculaire puissamment développée. Pami les auteurs qui ont étudié $C$. strumosa, personne ne leur avait trouvé de spicule. Il en résulte que $C$. strumosa doit être reconnu comme appartenant au genre Eucoleus.

Ainsi, actuellement, nous connaissons trois espèces du genre Eucoleus chez les Oiseaux. Une description sommaire de ces parasites est donnée ci-dessous.

Sous-ordre..... Trichocephalala Skrjabin et Schultz, 1926

Famille........ Trichocephalidæe Baird, 1853

Genre.......... Eucoleus Duj., 1845

\section{Eucoleus corvicola Wassilkowa, 1930}

Nous avons constaté 4 mâles et 4 femelles de ce genre chez des Oiseaux de la famille des Corvidés : Pica pica, Corvus cornix, Garrulus glandarius.

Annales de Parasitologie, T. VIII, $\mathrm{N}^{\circ} 6 .-1^{\text {er }}$ décembre $1930, \mathrm{p} .619-623$. 
Habitat : œsophage.

Distribution géographique : gouvernement de Sévéro-Dvinsk (U. R.S. S.)

Des représentants de ce genre ont été aussi trouvés par N. Shikobalova chez Corvus monedula et Corvus cornix, tous deux provenant d'Arménie.

Description. - Le mâle a un corps filiforme et transparent, atténué graduellement vers les deux extrémités. La longueur totale du corps est de $12 \mathrm{~mm}$., 77-15 mm., 63, avec un maximum de largeur de $100 \mu$. Longueur de l'œsophage : 410 à $480 \mu$. L'extrémité caudale en forme de bourse est munie de deux grosses papilles. Pas de spicule. La gaine spiculaire, longue de 420-520 $\mu$ est garnie de petites épines irrégulièrement disposées le long du trajet: sur l'extrémité proximale, les épines sont plus petites et plus serrées, ensuite vient une portion où elles sont plus grandes mais moins serrées, après quoi leur nombre augmente considérablement pour devenir insignifiant à l'extrémité distale. La largeur de la gaine spiculaire est inégale : 200 à $240 \mu$ à l'extrémité proximale et $120 \mu$ à l'extrémité distale : là où les épines sont rares, la gaine se rétrécit jusqu'à $8 \mu$.

Le corps de la femelle est jaunâtre. Graduellement effilé vers les extrémités, il atteint une longueur de $14 \mathrm{~mm}$., 16 à $18 \mathrm{~mm}$., 57 avec une largeur maxima à $150 \mu$. La longueur de l'osophage est de $3 \mathrm{~mm} ., 95-4 \mathrm{~mm} ., 82$. La vulve se trouve au tiers antérieur du corps, à une distance de $4 \mathrm{~mm}$., 07 à $5 \mathrm{~mm}$., 34 de l'extrémité céphalique. CEufs typiques en forme de tonneaux $(60 \mu, 30 \mu)$. L'extrémité caudale, assez large, est munie de papilles coniques. Les stries cuticulaires, couvertes de petites saillies verruqueuses, sont bien visibles, tout comme chez les mâles. L'anus est terminal.

\section{Eucoleus laricola Wassilkowa, 1930}

Cette seconde espèce du genre Eucoleus a été rencontrée chez Larus ridibundus, L. canus, L. argentatus, Sterna hirundo, Hydrochelidon nigra.

Habitat : œsophage.

Distribution géographique : Sibérie de l'Ouest et Extrême-Orient de I'U. R.S.S.

Description. - Le corps filiforme du màle est long de $10 \mathrm{~mm}$., 365 à $13 \mathrm{~mm}$., 142, avec au milieu, une largeur de $89 \mu$. La longueur de l'œsophage oscille entre $2 \mathrm{~mm}$., 538 et $3 \mathrm{~mm}$., 331 . La cuticule forme à l'extrémité caudale des saillies lobulaires : deux pairès du côté 
antérieur et une paire du côté postérieur, toutes munies d'une paire de papilles et le tout recouvert par la bourse campanuliforme. Pas de spicule. La gaine spiculaire atteint $465 \mu-595 \mu$ de long. Sur toute son étendue, elle est recouverte de petites épines courtes et obtuses à l'extrémité proximale, pointues et longues de $14 \mu$ partout ailleurs. Sur le tiers antérieur de la gaine, ces épines sont serrées; sur les deux autres tiers, elle le sont moins et leur répartition y est fort régulière. La largeur de la gaine spiculaire est à l'extrémité proximale de $120 \mu-200 \mu$ et l'extrémité dorsale est légèrement atténuée.

La longueur totale de la femelle varie entre $14 \mathrm{~mm}$., 622 et $17 \mathrm{~mm}$., 481, la largeur maxima est de $129 \mu$. L'œesophage est long de $3 \mathrm{~mm}$., 05-3 mm., 88. La vulve est placée dans le tiers antérieur du corps ; vers l'orifice elle forme un renflement simulant des lèvres.

CEufs longs de $56 \mu-60 \mu$, sur une largeur de 24 à $28 \mu$. L'anus est terminal. Les stries cuticulaires, verruqueuses, sont surtout bien visibles chez les femelles; chez les mâles elles sont moins nettes.

\section{Eucoleus strumosa (Reibisch, 1893) Gouchanskaja}

Jusqu'à présent ce parasite n’a été décrit que chez Phasianus colchicus et Gallus gallus domest : nous avons pu le constater chez Lyrurus tetrix L.

- Habitat : jabot et œsophage (sous l'épithélium).

Distribution géographique : Allemagne, Italie, Roumanie, Brésil et U. R. S. S. (Sibérie).

La description qui suit est fondée sur l'étude des exemplaires que nous obtînmes chez des Lyrurus tetrix. L'invasion a été constatée chez 9 tétras chez lesquels on a trouvé 27 femelles et 4 mâles.

Les nématodes, filiformes, de couleur jaunâtre, sont fortement atténués vers l'extrémité céphalique et légèrement atténués vers l'extrémité caudale. L'extrémité céphalique est munie d'une expansion ovale de la cuticule fort caractéristique. L'orifice buccal est simple. L'œsophage est formé d'une chaînette de cellules cylindriques' détachées. La lumière de l'œsophage passe par le centre de chaque cellule.

Le corps du mâle mesure $23 \mathrm{~mm} ., 17$. La longueur de l'œsophage est de $3 \mathrm{~mm}$., $15-3 \mathrm{~mm}$., 65. La largeur de l'extrémité céphalique, à l'exception de l'expansion cuticulaire, est de $17 \mu$ et de 46 à $49 \mu$ y compris l'expansion. La largeur du corps au niveau où l'œsophage se termine est de $81 \mu$ et celle de l'extrémité postérieure de $37 \mu$. L'extrémité caudale se termine par deux lobes latéraux, arrondis. Le pore excréteur s'ouvre plutôt ventralement. Pas de spicule. La 
gaine spiculaire est munie d'épines dont la disposition est la suivante : l'extrémité proximale porte de petites épines sur une étendue de $101 \mu$ ensuite elles augmentent de dimensions et un espace de $197 \mu$ est couvert de grosses épines dont les extrémités sont tournées vers l'extrémité proximale. La portion suivante, de $275 \mu$ de long, est dénuée d'épines, puis en vient une de $950 \mu$ qui en porte de grandes et de là, vers l'extrémité distale, les dimensions des épines diminuent graduellement.

La longueur totale du corps de la femelle est de $34 \mathrm{~mm} .92$ à $37 \mathrm{~mm}$., 63. La largeur de l'extrémité céphalique sans l'expansion cuticulaire est de $17 \mu$ et avec l'expansion de 43 à $46 \mu$. La largeur du corps dans la portion postérieure de l'œsophage atteint $124 \mu$. La largeur de l'extrémité postérieure du corps est de $37 \mu$. La vulve est à une distance de $29 \mu$ de l'extrémité de l'œsophage et à une distance de 5 mm., 21-5 mm., 409 de l'extrémité céphalique. Les œufs, oblongs, quelque peu asymétriques, portent de petits bouchons aux pôles. Dimensions des œufs : 55 à $58 \mu$ de longueur sur 25 à $26 \mu$ de largeur. L'anus est presque terminal.

Les chiffres, donnés par Ciurea pour les parasites qu'il constata dans le jabot des poules domestiques, correspondent presque entièrement avec ceux que nous venons d'indiquer. Ciurea rattache le parasite qu'il a décrit à Trichosoma strumosa Reibisch, 1893. Travassos le considère comme appartenant au sous-genre Thominx en le désignant comme Capillaria (Thominx) strumosa parce que la gaine spiculaire est couverte d'épines. Aucun auteur n'a donné les dimensions du spicule. Ainsi Ciurea, en citant l'ouvrage de Reibisch, dit : "La gaine spiculaire est couverte d'épines délicates " et ne fait même pas mention du spicule. Evidemment Reibesch, qui a été le premier à décrire ce genre, n'avait pas trouvé de spicule chez le màle. Exposant ses recherches Ciurea dit : "Uber das Spiculum kann ich leider nicht mehr berichten als dass es 0,004 breit ist da ich dasselbe trotz vieler Mühe weder in seiner Scheide verfolgen noch einen Teil aus derselben herausziehen kenne (1). " Sur le dessin donné par cet auteur (extrémité caudale du mâle) on voit distinctement la gaine spiculaire, étirée en dehors et couverte d'épines, mais le spicule manque. La largeur du spicule dont Ciurea parle n'est sûrement autre chose que la lumière de la gaine spiculaire. Le dessin de l'extrémité caudale du mâle, de même que celui de l'extrémité céphalique, reproduits par Ciurea, traduisent parfaitement ce que nous avons constaté chez notre parasite. Les dimensions de tous les organes sont aussi identiques.

(1) Ciurea. - Nematoden aus den Pharynx und Esophagus des Haushuhnes. Ztsch. f. Infektionsk, $u$, parasitäre Krankh. der Haustiere, XV, 1914. 
Nous basant sur toutes ces données, nous croyons possible de transporter Capillaria (Thominx) strumosa du sous-genre Thominx au genre Eucoleus.

\section{RÉsumé}

1. Actuellement on doit rapporter au genre Eucoleus Duj., 1845, les 6 espèces suivantes :

1. Eucoleus aerophilus Creplin, 1839.

2. Encoleus tenuis Duj., 1845.

3. Eucoleus baskakowi Schultz, 1929.

4. Eucoleus corvicola Wassilkowa, 1930.

5. Eucoleus laricola Wassilkowa, 1930.

6. Eucoleus strumosus (Reibisch, 1893) Gouschanskaja, 1930.

2. La diagnose du genre Eucoleus Duj., 1845, doit être modifiée en raison de ce que ses représentants peuvent vivre en parasites, non seulement chez les Mammifères, mais aussi chez les Oiseaux et qu'ils peuvent être localisés aussi bien dans les voies respiratoires que dans le tube digestif.

3. Il est fort probable qu'une révision ultérieure des Capillarinæe contribuera à faire transporter dans le genre Eucoleus Duj., 1845 une partie des espèces rapportées au genre Capillaria Zeder, 1800.

4. Il est indispensable de revoir l'espèce Capillaria concerta et de donner des indices différentiels bien préeis pour distinguer les mâles et les femelles de cette espèce d'avec ceux d'Eucoleus corvicola et Eucoleus laricola.

Nous ne voulons pas finir cet exposé sans exprimer ici au professeur K. Skrjabin notre profonde reconnaissance pour les précieuses indications qu'il a bien voulu nous donner au cours de nos recherches.

Inslitul central d'helminthologie de Moscou. 